\title{
The Optimal Therapeutic Strategy for Patients with EGFR-Mutated Non-Small Cell Lung Cancer with Brain Metastasis: A Real-World Study from Taiwan
}

\section{Wen-Chien Cheng}

China Medical University Hospital

\section{Yi-Cheng Shen}

China Medical University Hospital

Chun-Ru Chien

China Medical University

Wei-Chih Liao

China Medical University Hospital

\section{Chia-Hung Chen}

China Medical University Hospital

\section{Te-Chun Hsia}

China Medical University Hospital

Chih-Yeh Tu

China Medical University Hospital

Hung-Jen Chen ( $\nabla$ redman0127@gmail.com )

China Medical University Hospital

\section{Research Article}

Keywords: Optimal Therapeutic, Brain Metastasis, crucial

Posted Date: December 23rd, 2021

DOl: https://doi.org/10.21203/rs.3.rs-1183444/v1

License: (1) (1) This work is licensed under a Creative Commons Attribution 4.0 International License. Read Full License 


\section{Abstract}

Objective

The treatment options for epidermal growth factor receptor (EGFR)-mutated non-small-cell lung cancer (NSCLC) with brain metastases (BMs) include EGFR-tyrosine kinase inhibitors (TKIs), stereotactic radiosurgery (SRS), whole-brain radiotherapy (WBRT), brain surgery (BS), and anti-angiogenesis therapy. As treatment options evolve, the redefinition of optimal treatment strategies for improving survival is crucial.

Methods

150 EGFR-mutant NSCLC patients with BMs who received first- or second-generation EGFR-TKIs as firstline treatment between January 2012 and October 2019 were included in this analysis.

Results

After multivariate analysis, patients with graded prognostic assessments for lung cancer based on molecular markers (Lung-mol GPA) $\geq 3$ (Hazard ratio (HR): 0.538, 95\% Confidence Interval (Cl): 0.350.83 ); who received afatinib or erlotinib as first-line treatment (HR:0.521, 95\% Cl: 0.33-0.82); underwent SRS therapy (HR:0.531, 95\%Cl: 0.32-0.87); or were sequentially treated with osimertinib (HR:0.400, 95\%Cl: 0.23-0.71) were associated with improved overall survival (OS). Furthermore, SRS plus EGFR-TKI provided more OS benefits in patients with Lung-mol GPA $\geq 3$ compared with EGFR-TKI alone in our patient cohort (44.9 vs. 26.7 months, $p=0.005$ ). The OS among patients who received sequential osimertinib therapy was significantly longer than those without osimertinib treatment ( 43.5 vs. 24.3 months, $p<0.001$ ), regardless of T790 mutation status (positive vs. negative/unknown: 40.4 vs. 54.6 months, $p=0.093$ ).

Conclusions

The study demonstrated that EGFR-mutant NSCLC patients with BMs could be precisely treated with SRS according to Lung-mol GPA $\geq 3$. Sequential osimertinib was associated with prolonged survival, regardless of T790M status.

\section{Introduction}

Non-small cell lung cancer (NSCLC) accounts for $85 \%$ of all lung cancers, and brain metastases (BMs) are a frequent complication of NSCLC. Approximately $10 \%-20 \%$ of NSCLC patients had BMs at initial diagnosis, and approximately $20 \%-40 \%$ of NSCLC patients developed BMs during treatment.[1] Epidermal growth factor receptor (EGFR) mutation is associated with approximately $40 \%-60 \%$ of Asian NSCLC patients and $10 \%$ of Western patients. [2] The incidence of BMs is higher in patients with EGFR mutations than in those with wild-type EGFR.[3] Three generations of EGFR-tyrosine kinase inhibitors (EGFR-TKIs) have been approved for use in first-line treatment for EGFR-mutant NSCLC patients with BMs.[4-9] The penetration rates to cerebrospinal fluid (CSF) from plasma for gefitinib, erlotinib, afatinib, and osimertinib 
are $1.13 \%, 2.77 \%, 2.5 \%$, and 5\%, respectively.[10-12] The third-generation EGFR-TKI, osimertinib, had significantly better progression-free survival (PFS), overall survival (OS), and BM response than firstgeneration EGFR-TKIs.[13, 14]

Despite these results, osimertinib is limited for use as a first-line strategy in clinical practice in many countries due to its high price. In addition, no OS benefit of osimertinib compared with gefitinib, erlotinib, or afatinib v was observed in patients of Asian descent.[7, 15-17] Therefore, first- or second-generation EGFR-TKIs remain the first-line treatment in many Asian patients with a new diagnosis of EGFR-mutant NSCLC.

Because the measurable CSF concentration for first- or second-generation EFGR-TKIs in CSF is much lower than that for osimertinib, the combination of first- or second-generation EGFR-TKI and local therapy, such as stereotactic radiosurgery (SRS), whole-brain radiotherapy (WBRT), or brain surgery (BS), had been investigated as an aggressive therapy in selective EGFR mutant NSCLC patients with BMs. A metaanalysis of 1,465 patients demonstrated that the combination of brain radiotherapy (RT) and EGFR-TKI had better survival outcomes, especially in cases of SRS.[18]

The disease-specific graded prognostic assessment (DS-GPA) has previously been used for RT treatment decisions.[19] Superduto et al. have upgraded from DS-GPA to Lung-mol GPA, which includes the EGFR and $A L K$ mutation status.[20] However, few studies examining the efficacy of EGFR-TKIs combined with local therapy have addressed the Lung-mol GPA.[21]

Combination systemic therapy using anti-angiogenesis agents and EGFR-TKIs also provided better intracranial control rates, longer times to intracranial progression, and fewer new BMs than EGFR-TKIs alone.[22, 23] Clinical trials (JO25567, NEJ026, and RELAY) also found that erlotinib plus vascular endothelial growth factor (VEGF) or vascular endothelial growth factor receptor (VEGFR) inhibitor significantly prolonged PFS among patients with EGFR-mutant NSCLC.[24-26]

Most cancers will progress after first-line treatment with first- or second-generation EGFT-TKIs. Sequential treatment utilizing osimertinib as the second-line treatment has shown promising results, particularly among those who develop the T790M mutation.[27] However, our previous study showed that central nervous system (CNS) progression was inversely correlated with the presence of the T790M mutation. [28] This outcome may be associated with the difficulty obtaining a biopsy for CNS lesions, the recognized mechanisms of pharmacokinetic resistance. and the poor CSF-to-plasma ratio reported for first- and second-generation EGFR-TKIs.[29]

The identification of suitable candidates for treatment with local therapy or anti-angiogenesis agents in combination with EGFR-TKIs and the assessment of the sequential treatment strategy using osimertinib as a second-line treatment among patients with CNS progression remain necessary. We conducted this retrospective study with real-world data to determine the optimal treatment strategy for EGFR-mutant NSCLC patients with BMs, which may help prolong survival. 


\section{Materials And Methods}

\section{Study participants}

We conducted a retrospective study to analyze EGFR-mutant adenocarcinoma patients with initial BMs who started EGFR-TKI (gefitinib, erlotinib, or afatinib) as first-line therapy between January 2012 and October 2019 at China Medical University Hospital. Patients who were diagnosed with BMs, confirmed by brain magnetic resonance imaging (MRI) or computed tomography (CT), prior to initiating EGFR-TKI therapy were included. The exclusion criteria included patients with insufficient data for analysis; those who received treatment for less than three months; or those without EGFR mutation. The Institutional Review Board of China Medical University Hospital approved this study (CMUH 110-REC3-110), and informed consent was waived due to the observational and retrospective study design.

\section{Clinical data acquisition}

The following information was extracted from electronic health records: age, sex, smoking history, Eastern Cooperative Oncology Group performance status (ECOG-PS), the Karnofsky's index of performance status (KPS), type of sensitizing EGFR mutation, EGFR-TKI treatment, PFS, the number and maximum size of brain tumors, baseline metastatic site, Lung-mol GPA score,[20] treatment strategies for BMs, T790M status, and the sequence osimertinib treatment. PFS was defined as the period from the initiation date of EGFR-TKI treatment to the date of radiologic or clinical evidence of progression or death. OS was defined as the time from lung cancer diagnosis to death due to any cause. The Lung-mol GPA score included age, KPS, number of BMs, presence of extracranial metastasis, and gene mutation status. [20] Additional local therapies for BMs included radiation therapy, such as WBRT or SRS, and craniotomy with brain tumor removal. Anti-angiogenesis therapy, including bevacizumab or ramucirumab, was added according to the physician's assessment.

\section{Statistical analyses}

Continuous variables are presented as the mean and standard deviation (SD) or median and interquartile range (IQR; 25th and 75th percentiles). Categorical variables are expressed as percentages. Differences between continuous variables were compared using the Mann-Whitney $\mathrm{U}$ test or the independent ttest. Differences between two independent categorical variables were compared by the Chi-square test or Fisher's exact test. A receiver operating characteristic (ROC) curve was used to determine the cutoff value of the Lung-mol GPA. Univariate and multivariable Cox regression analyses were used to evaluate which factors are independently associated with prognosis among these patients. OS was estimated using the Kaplan-Meier method, and differences among different treatments were compared using the log-rank test. A $p$-value $<0.05$ was considered significant. All statistical analyses were analyzed using MedCalc for Windows version 18.10 (MedCalc Software, Ostend, Belgium).

\section{Results}




\section{Patient baseline characteristics}

From January 2012 to October 2019, 3,562 patients were diagnosed with lung cancer, and 812 patients with stage IIIB-IV lung adenocarcinoma received EGFR-TKI as first-line therapy. A total of 150 patients with initial BMs were enrolled in this study after the exclusion criteria were applied. Among these patients, 37 (37/150, 24.6\%) received gefitinib, 76 (76/150, 50.6\%) received erlotinib, and 37(37/150, 24.6\%) received afatinib as first-line therapy (Figure 1). The baseline characteristics of all patients are shown in Table 1. The cutoff values for the Lung-mol GPA score in our cohort were determined based on the area under the ROC curve. Scores of 3 or above were defined as indicators of good prognosis, which resulted in values for the area under the ROC curve, sensitivity, and specificity of $0.626,0.61$, and 0.58 , respectively. A higher proportion of patients who received afatinib were younger than 65 years and presented with better ECOG-PS. The proportion of patients with exon 21 L858R mutation was higher among those patients who received erlotinib. No significant differences in sex, smoking status, the proportion of the neurologic symptoms, the maximum size of BMs, the number of patients with leptomeningeal metastasis, or the proportion of patients with Lung-mol GPA $\geq 3$ were observed among these three EGFR-TKI treatment groups.

\section{Treatment strategies and response assessment}

After a median follow-up of 40.4 months (range: 33.7-49.7 months), 97 of 150 patients had died. As shown in Table 1, the use of WBRT was lower, and the combination of anti-angiogenic regimens was higher in the erlotinib group. Significantly longer PFS was noted among those patients who received afatinib as first-line therapy (gefitinib vs. erlotinib vs. afatinib: 8.4 vs. 10.6 vs. 12.1 months, $p=0.042$ ). After the failure of first-line EGFR-TKIs, 36 patients (36/150, 24\%) were diagnosed with isolated CNS relapse; 74 patients $(74 / 150,49.3 \%)$ did not receive T790M mutation testing (unknown group); 46 patients (46/150, 30.6\%) did not have the T790M mutation (negative group); and 30 patients (30/150, $20 \%$ ) were diagnosed with T790M mutation (T790M-positive group). A total of 42 patients received osimertinib as a later-line treatment. Patients who received erlotinib as first-line treatment had a higher rate of sequential treatment with osimertinib (gefitinib vs. erlotinib vs. afatinib: $8.1 \%$ vs. $42.1 \%$ vs. 18.9 $\%, p<0.001 ;$ Table 1$)$.

\section{Clinical factors associated with survival outcomes}

We performed univariate and multivariate analyses of clinical factors predicting survival outcomes in EGFR-mutant NSCLC patients with BMs (Table 2). Significantly longer OS was noted in patients with Lung-mol GPA $\geq 3$ (hazard ratio [HR]: $0.538, p=0.005$ ). Afatinib or Erlotinib as first-line treatment significantly reduced mortality compared with gefitinib (HR: $0.521, p=0.004$ ). The addition of local therapy with SRS provided patients with better outcomes (HR: 0.531, $p=0.014$ ), and patients treated with EGFR-TKI plus SRS had increased median OS than those without SRS (39.4 vs. 24.8 months; $p=0.002$; Figure 2A). Patients were divided into two groups to identify potential differences in the benefits of additional treatment (Lung-mol GPA $\geq 3$ and Lung-mol GPA $<3$ ). The median OS for patients with Lungmol GPA $\geq 3$ who received EGFR-TKI plus SRS was longer than for those treated with EGFR-TKI without 
SRS (44.9 vs. 26.7 months, $p=0.005$; Figure 2B). However, no significant difference in OS was observed between patients with Lung-mol GPA $<3$ who received EGFR-TKI plus SRS and those who received EGFRTKI without SRS (30.2 vs. 22.2 months, $p=0.309$; Figure 2C). As shown in Table 2, patients who received anti-angiogenesis agents appeared to present with longer OS than those without anti-angiogenesis treatment, based on the outcomes of the univariate analysis (HR: 0.454, $p=0.044$ ). However, no significant difference in OS was observed among patients treated with and without anti-angiogenesis agents in the multivariate analysis (HR: 0.579, $p=0.169$ ).

The OS in patients who received sequential osimertinib therapy was significantly longer than in those without osimertinib treatment (43.5 vs. 24.3 months, $p<0.001$; Figure $3 \mathrm{~A}$ ). Among those who received osimertinib, no difference in OS was observed between patients with and without/unknown T790M (40.4 vs. 54.6 months, $p=0.093$; Figure 3B). Furthermore, as shown in Figure 4, significantly longer survival was observed in patients who received sequential osimertinib therapy, regardless of the use of additional local brain therapy.

\section{Discussion}

To our knowledge, our study is the first study to examine the effects of Lung-mol GPA and different treatment strategies on survival in NSCLC EGFR-mutant patients with BMs. We found a significantly longer OS in NSCLC EGFR-mutant patients with BMs who received afatinib or erlotinib as first-line treatment in combination with SRS. EGFR-TKI plus SRS provided more OS benefits for patients with Lungmol GPA $\geq 3$. Sequential osimertinib therapy provided OS benefits regardless of the status of T790M mutation or the addition of local brain control.

Monotherapy using first-generation of EGFR-TKIs can result in a $67 \%-88 \%$ intracranial objective response rate in EGFR-TKI-naïve EGFR-mutant NSCLC patients.[5, 8, 9] Several retrospective studies indicated that erlotinib is more effective than gefitinib in treating BMs due to higher levels of drug in the CSF.[10, 30] Jung et al. reported that afatinib showed a superior tendency for CNS-PFS compared with gefitinib or erlotinib.[31] Our study reported that the initial use of afatinib or erlotinib was an independent prognostic factor for OS, which might be consistent with these studies (Table 2).

Although EGFR-TKI monotherapy provides an acceptable intracranial response in EGFR-mutant NSCLC patients, additional local treatments were investigated as aggressive treatment options to prolong intracranial control. Two meta-analysis studies reported that cranial RT (WBRT or SRS) plus TKI had higher intracranial PFS and OS than TKI therapy alone.[18,32] However, these studies have rarely reported the benefits of cranial RT in NSCLC patients based on Lung-mol GPA scores. Magnuson et al. indicated that patients who received upfront SRS had longer OS than those treated with WBRT or those who received EGFR-TKI followed by RT. The survival benefit was more evident in patients with DS-GPA 2-4 than in those with DS-GPA 0-1.5.[21] The current study also indicated that patients with Lung-mol GPA $\geq$ 3 who received SRS had longer OS than those who did not receive SRS (Figure 2B). The inconsistent cutoff value of Lung-mol GPA may be related to the different study cohort. Therefore, in NSCLC EGFR- 
mutant patients with BMs, SRS provided better control in patients without extracranial metastases (Lungmol GPA $\geq 3$ ). However, the addition of WBRT did not result in an OS benefit (Table 2), which was not consistent with the results reported by Wang et al., [32] which may be due to the influence of osimertinib on WBRT.

The dominant status of cranial RT for the treatment of EGFR-mutant BMs has been challenged by the wide use of newer-generation targeted therapies.[33] A phase II study was conducted to evaluate the efficacy of osimertinib in patients with previously untreated BMs to avoid brain RT.[34] Our study showed that patients treated with sequential osimertinib therapy had similar OS regardless of the use of additional local therapy (Figure 4), indicating that treatment with osimertinib could reduce the use of local therapy and avoid associated side effects. Furthermore, the current study indicated that patients with CNS-progressed disease after first-line EGFR-TKI treatment who received osimertinib as sequential treatment had OS benefits regardless of T790M status. Lee et al. also reported an improvement in OS for patients who developed leptomeningeal metastases following first- or second-generation EGFR-TKI failure and were treated with subsequent osimertinib, regardless of T790M mutational status.[35] Poor CNS penetration of first- and second-generation EGFR-TKIs is associated with pharmacokinetic resistance.[36] The superior penetration of osimertinib through the blood-brain barrier (BBB) may explain this phenomenon.

Several limitations should be noted for this retrospective study. First, the choice of EGFR-TKI treatment was made by the clinical physician; therefore, the number of patients in the erlotinib group was relatively larger than the numbers in the other two groups, which may be influenced by previous studies showing a higher BBB penetration rate for erlotinib. Therefore, multivariate analysis was performed to minimize potential bias. Second, the current study did not provide intracranial PFS due to a lack of regular followup brain MRI data. Third, 74 (49.3\%) patients in our cohort did not receive T790M testing due to difficulties obtaining a second biopsy, especially among the $36(24 \%)$ patients with isolated CNS progression first-line EGFR-TKI failure. Fourth, financial difficulties were identified among patients treated with anti-angiogenesis because these medicines are not supported by the national health insurance program in Taiwan. Finally, the number of patients treated with anti-angiogenesis therapy was too small to achieve significance, and the effects of anti-angiogenesis therapy on outcomes warrant further study. In spite of these limitations, our study identified optimal treatment strategies for EGFR-mutant patients with BM in the era of new generations of EGFR-TKIs.

\section{Conclusion}

In summary, this study demonstrated that a favorable survival prognosis was identified for EGFR-mutant NSCLC patients with BMs with Lung-mol GPA $\geq 3$ who were treated with afatinib or erlotinib in combination with SRS. Sequential osimertinib therapy may be used in place of local brain treatment, regardless of T790M status.

\section{Abbreviations}


BBB: blood-brain barrier; BS: brain surgery; BMs: brain metastases; CSF: cerebrospinal fluid; CNS: central nervous system; Del19: EGFR exon 19 deletion; ECOG-PS: Eastern Cooperative Oncology Group performance status; EGFR: epidermal growth factor receptor; EGFR-TKI: epidermal growth factor receptor tyrosine kinase inhibitor; GPA: graded prognostic assessment; IQR: interquartile range; KPS: Karnofsky performance status; NSCLC: non-small-cell lung cancer; OS: overall survival; PFS: progression-free survival; RT: radiotherapy; SRS: stereotactic radiosurgery; TKI: tyrosine kinase inhibitor; VEGF: vascular endothelial growth factor; VEGFR: vascular endothelial growth factor receptor; WBRT: whole-brain radiotherapy

\section{Declarations}

\section{Acknowledgments}

The authors wish to thank Chia-Ing Li for her technical help with the statistical analysis.

\section{Funding}

Not applicable.

\section{Availability of data and materials}

Not applicable.

\section{Authors' contributions}

WCC and HJC participated in study conception and design. WCC, YCS, CRC, WCL, CHC, TCH, CYT, and HJC participated in data acquisition. WCC and HJC participated in data analysis and interpretation. WCC and YCS drafted the manuscript, with all authors revising it critically for intellectual content. All authors have read and approved the final version of the manuscript.

\section{Competing interests}

No conflicts exist for the specified authors.

\section{Consent for publication}

All authors have reviewed and approved the manuscript for publication.

\section{Ethics approval and consent to participate}

The Investigational Review Board approved this review at China Medical University Hospital (CMUH110REC3-110). The requirement for individual patient consent was deemed unnecessary because of the retrospective study design.

\section{Author details}


${ }^{1}$ Division of Pulmonary and Critical Care, Department of Internal Medicine, China Medical University Hospital, Taichung, Taiwan.

${ }^{2}$ School of Medicine, College of Medicine, China Medical University, Taichung, Taiwan

${ }^{3}$ Department of Life Science, National Chung Hsing University, Taichung, Taiwan

${ }^{4}$ Ph.D. Program in Translational Medicine, National Chung Hsing University, Taichung, Taiwan

${ }^{5}$ Rong Hsing Research Center for Translational Medicine, National Chung Hsing University, Taichung, Taiwan

${ }^{6}$ Department of Radiation Oncology, China Medical University Hospital, Taichung, Taiwan

Clinical trial registry number

Not applicable

\section{References}

1. Arrieta O, Saavedra-Perez D, Kuri R, Aviles-Salas A, Martinez L, Mendoza-Posada D, Castillo P, Astorga A, Guzman E, De la Garza J: Brain metastasis development and poor survival associated with carcinoembryonic antigen (CEA) level in advanced non-small cell lung cancer: a prospective analysis. BMC Cancer 2009, 9:119.

2. Pao W, Girard N: New driver mutations in non-small-cell lung cancer. Lancet Oncol 2011, 12(2):175-180.

3. Rangachari D, Yamaguchi N, VanderLaan PA, Folch E, Mahadevan A, Floyd SR, Uhlmann EJ, Wong ET, Dahlberg SE, Huberman MS et al: Brain metastases in patients with EGFR-mutated or ALK-rearranged non-small-cell lung cancers. Lung Cancer 2015, 88(1):108-111.

4. Mok TS, Wu YL, Thongprasert S, Yang CH, Chu DT, Saijo N, Sunpaweravong P, Han B, Margono B, Ichinose $Y$ et al: Gefitinib or carboplatin-paclitaxel in pulmonary adenocarcinoma. N Engl J Med 2009, 361(10):947-957.

5. Wu YL, Zhou C, Cheng Y, Lu S, Chen GY, Huang C, Huang YS, Yan HH, Ren S, Liu Y et al: Erlotinib as second-line treatment in patients with advanced non-small-cell lung cancer and asymptomatic brain metastases: a phase II study (CTONG-0803). Ann Oncol 2013, 24(4):993-999.

6. Wu YL, Zhou C, Hu CP, Feng J, Lu S, Huang Y, Li W, Hou M, Shi JH, Lee KY et al: Afatinib versus cisplatin plus gemcitabine for first-line treatment of Asian patients with advanced non-small-cell lung cancer harbouring EGFR mutations (LUX-Lung 6): an open-label, randomised phase 3 trial. Lancet Oncol 2014, 15(2):213-222. 
7. Cheng Y, He Y, Li W, Zhang HL, Zhou Q, Wang B, Liu C, Walding A, Saggese M, Huang X et al: Osimertinib Versus Comparator EGFR TKI as First-Line Treatment for EGFR-Mutated Advanced NSCLC: FLAURA China, A Randomized Study. Target Oncol 2021, 16(2):165-176.

8. Park SJ, Kim HT, Lee DH, Kim KP, Kim SW, Suh C, Lee JS: Efficacy of epidermal growth factor receptor tyrosine kinase inhibitors for brain metastasis in non-small cell lung cancer patients harboring either exon 19 or 21 mutation. Lung Cancer 2012, 77(3):556-560.

9. Iuchi T, Shingyoji M, Sakaida T, Hatano K, Nagano O, Itakura M, Kageyama H, Yokoi S, Hasegawa Y, Kawasaki $\mathrm{K}$ et al: Phase II trial of gefitinib alone without radiation therapy for Japanese patients with brain metastases from EGFR-mutant lung adenocarcinoma. Lung Cancer 2013, 82(2):282-287.

10. Togashi Y, Masago K, Masuda S, Mizuno T, Fukudo M, Ikemi Y, Sakamori Y, Nagai H, Kim YH, Katsura $\mathrm{T}$ et al: Cerebrospinal fluid concentration of gefitinib and erlotinib in patients with non-small cell lung cancer. Cancer Chemother Pharmacol 2012, 70(3):399-405.

11. Tamiya A, Tamiya M, Nishihara T, Shiroyama T, Nakao K, Tsuji T, Takeuchi N, Isa SI, Omachi N, Okamoto $\mathrm{N}$ et al: Cerebrospinal Fluid Penetration Rate and Efficacy of Afatinib in Patients with EGFR Mutation-positive Non-small Cell Lung Cancer with Leptomeningeal Carcinomatosis: A Multicenter Prospective Study. Anticancer Res 2017, 37(8):4177-4182.

12. Yang JCH, Kim SW, Kim DW, Lee JS, Cho BC, Ahn JS, Lee DH, Kim TM, Goldman JW, Natale RB et al: Osimertinib in Patients With Epidermal Growth Factor Receptor Mutation-Positive Non-Small-Cell Lung Cancer and Leptomeningeal Metastases: The BLOOM Study. J Clin Onco/2020, 38(6):538-547.

13. Soria JC, Ohe Y, Vansteenkiste J, Reungwetwattana T, Chewaskulyong B, Lee KH, Dechaphunkul A, Imamura F, Nogami N, Kurata T et al: Osimertinib in Untreated EGFR-Mutated Advanced Non-Small-Cell Lung Cancer. N Engl J Med 2018, 378(2):113-125.

14. Reungwetwattana T, Nakagawa K, Cho BC, Cobo M, Cho EK, Bertolini A, Bohnet S, Zhou C, Lee KH, Nogami N et al: CNS Response to Osimertinib Versus Standard Epidermal Growth Factor Receptor Tyrosine Kinase Inhibitors in Patients With Untreated EGFR-Mutated Advanced Non-Small-Cell Lung Cancer. J Clin Oncol 2018:JCO2018783118.

15. Ohe Y, Imamura F, Nogami N, Okamoto I, Kurata T, Kato T, Sugawara S, Ramalingam SS, Uchida H, Hodge $\mathrm{R}$ et al: Osimertinib versus standard-of-care EGFR-TKI as first-line treatment for EGFRm advanced NSCLC: FLAURA Japanese subset. Jpn J Clin Oncol 2019, 49(1):29-36.

16. Cho BC, Chewaskulyong B, Lee KH, Dechaphunkul A, Sriuranpong V, Imamura F, Nogami N, Kurata T, Okamoto I, Zhou C et al: Osimertinib versus Standard of Care EGFR TKI as First-Line Treatment in Patients with EGFRm Advanced NSCLC: FLAURA Asian Subset. J Thorac Oncol 2019, 14(1):99-106. 
17. Ito K, Morise M, Wakuda K, Hataji O, Shimokawaji T, Takahashi K, Furuya N, Takeyama Y, Goto Y, Abe $\mathrm{T}$ et al: A multicenter cohort study of osimertinib compared with afatinib as first-line treatment for EGFR-mutated non-small-cell lung cancer from practical dataset: CJLSG1903. ESMO Open 2021, 6(3):100115.

18. Du XJ, Pan SM, Lai SZ, Xu XN, Deng ML, Wang XH, Yao DC, Wu SX: Upfront Cranial Radiotherapy vs. EGFR Tyrosine Kinase Inhibitors Alone for the Treatment of Brain Metastases From Non-small-cell Lung Cancer: A Meta-Analysis of 1465 Patients. Front Oncol 2018, 8:603.

19. Sperduto PW, Chao ST, Sneed PK, Luo X, Suh J, Roberge D, Bhatt A, Jensen AW, Brown PD, Shih $\mathrm{H}$ et al: Diagnosis-specific prognostic factors, indexes, and treatment outcomes for patients with newly diagnosed brain metastases: a multi-institutional analysis of 4,259 patients. Int J Radiat Oncol Biol Phys 2010, 77(3):655-661.

20. Sperduto PW, Yang TJ, Beal K, Pan H, Brown PD, Bangdiwala A, Shanley R, Yeh N, Gaspar LE, Braunstein S et al: Estimating Survival in Patients With Lung Cancer and Brain Metastases: An Update of the Graded Prognostic Assessment for Lung Cancer Using Molecular Markers (Lung-molGPA). JAMA Oncol 2017, 3(6):827-831.

21. Magnuson WJ, Lester-Coll NH, Wu AJ, Yang TJ, Lockney NA, Gerber NK, Beal K, Amini A, Patil T, Kavanagh BD et al: Management of Brain Metastases in Tyrosine Kinase Inhibitor-Naive Epidermal Growth Factor Receptor-Mutant Non-Small-Cell Lung Cancer: A Retrospective Multi-Institutional Analysis. J Clin Oncol 2017, 35(10):1070-1077.

22. Feng PH, Chen KY, Huang YC, Luo CS, Wu SM, Chen TT, Lee CN, Yeh CT, Chuang HC, Han CL et al: Bevacizumab Reduces S100A9-Positive MDSCs Linked to Intracranial Control in Patients with EGFRMutant Lung Adenocarcinoma. J Thorac Oncol 2018, 13(7):958-967.

23. Jiang T, Zhang Y, Li X, Zhao C, Chen X, Su C, Ren S, Yang N, Zhou C: EGFR-TKIs plus bevacizumab demonstrated survival benefit than EGFR-TKIs alone in patients with EGFR-mutant NSCLC and multiple brain metastases. Eur J Cancer 2019, 121:98-108.

24. Saito H, Fukuhara T, Furuya N, Watanabe K, Sugawara S, Iwasawa S, Tsunezuka Y, Yamaguchi O, Okada $\mathrm{M}$, Yoshimori $\mathrm{K}$ et al: Erlotinib plus bevacizumab versus erlotinib alone in patients with EGFRpositive advanced non-squamous non-small-cell lung cancer (NEJ026): interim analysis of an open-label, randomised, multicentre, phase 3 trial. Lancet Oncol 2019, 20(5):625-635.

25. Seto T, Kato T, Nishio M, Goto K, Atagi S, Hosomi Y, Yamamoto N, Hida T, Maemondo M, Nakagawa $\mathrm{K}$ et al: Erlotinib alone or with bevacizumab as first-line therapy in patients with advanced nonsquamous non-small-cell lung cancer harbouring EGFR mutations (J025567): an open-label, randomised, multicentre, phase 2 study. Lancet Oncol 2014, 15(11):1236-1244. 
26. Nakagawa K, Garon EB, Seto T, Nishio M, Ponce Aix S, Paz-Ares L, Chiu CH, Park K, Novello S, Nadal E et al: Ramucirumab plus erlotinib in patients with untreated, EGFR-mutated, advanced non-smallcell lung cancer (RELAY): a randomised, double-blind, placebo-controlled, phase 3 trial. Lancet Oncol 2019, 20(12):1655-1669.

27. Mok TS, Wu YL, Ahn MJ, Garassino MC, Kim HR, Ramalingam SS, Shepherd FA, He Y, Akamatsu H, Theelen WS et al: Osimertinib or Platinum-Pemetrexed in EGFR T790M-Positive Lung Cancer. N Engl J Med 2017, 376(7):629-640.

28. Cheng WC, Hsia TC, Tu CY, Chen HJ: The Impact of Acquired EGFR T790M Mutation and EGFR Circulating Cell-Free DNA on Survival in Patients with Lung Adenocarcinoma Following EGFR-TKI Therapy. Onco Targets Ther 2020, 13:13425-13435.

29. Hata A, Katakami N, Yoshioka H, Kaji R, Masago K, Fujita S, Imai Y, Nishiyama A, Ishida T, Nishimura $Y$ et al: Spatiotemporal T790M Heterogeneity in Individual Patients with EGFR-Mutant NonSmall-Cell Lung Cancer after Acquired Resistance to EGFR-TKI. J Thorac Oncol 2015, 10(11):1553-1559.

30. Li MX, He H, Ruan ZH, Zhu YX, Li RQ, He X, Lan BH, Zhang ZM, Liu GD, Xiao HL et al: Central nervous system progression in advanced non-small cell lung cancer patients with EGFR mutations in response to first-line treatment with two EGFR-TKIs, gefitinib and erlotinib: a comparative study. BMC Cancer 2017, 17(1):245.

31. Jung HA, Woo SY, Lee SH, Ahn JS, Ahn MJ, Park K, Sun JM: The different central nervous system efficacy among gefitinib, erlotinib and afatinib in patients with epidermal growth factor receptor mutationpositive non-small cell lung cancer. Trans/ Lung Cancer Res 2020, 9(5):1749-1758.

32. Wang C, Lu X, Lyu Z, Bi N, Wang L: Comparison of up-front radiotherapy and TKI with TKI alone for NSCLC with brain metastases and EGFR mutation: A meta-analysis. Lung Cancer 2018, 122:94-99.

33. Sung S, Lee SW, Kwak YK, Kang JH, Hong SH, Kim YS: Intracranial control and survival outcome of tyrosine kinase inhibitor (TKI) alone versus TKI plus radiotherapy for brain metastasis of epidermal growth factor receptor-mutant non-small cell lung cancer. J Neurooncol 2018, 139(1):205-213.

34. Wakuda K, Yamaguchi H, Kenmotsu H, Fukuda M, Takeshita M, Suetsugu T, Kirita K, Ebi N, Hataji $\mathrm{O}$, Miura $\mathrm{S}$ et al: A phase II study of Osimertinib for patients with radiotherapy-naive CNS metastasis of non-small cell lung cancer: treatment rationale and protocol design of the OCEAN study (LOGIK 1603/WJOG 9116L). BMC Cancer 2020, 20(1):370.

35. Lee J, Choi Y, Han J, Park S, Jung HA, Su JM, Lee SH, Ahn JS, Park K, Ahn MJ: Osimertinib Improves Overall Survival in Patients With EGFR-Mutated NSCLC With Leptomeningeal Metastases Regardless of T790M Mutational Status. J Thorac Oncol 2020, 15(11):1758-1766. 
36. Park JH, Kim YJ, Lee JO, Lee KW, Kim JH, Bang SM, Chung JH, Kim JS, Lee JS: Clinical outcomes of leptomeningeal metastasis in patients with non-small cell lung cancer in the modern chemotherapy era. Lung Cancer 2012, 76(3):387-392.

\section{Tables}

Table 1. Patient Characteristics

\begin{tabular}{llllll} 
& All $(\mathbf{n = 1 5 0})$ & $\begin{array}{l}\text { Gefitinib }(\mathbf{n}= \\
\mathbf{3 7})\end{array}$ & $\begin{array}{l}\text { Erlotinib }(\mathbf{n}= \\
\mathbf{7 6})\end{array}$ & $\begin{array}{l}\text { Afatinib }(\mathbf{n}= \\
\mathbf{3 7})\end{array}$ & $\begin{array}{l}\boldsymbol{p} \text { - } \\
\text { value }\end{array}$ \\
\hline Age $\geq$ 65 years & $52(34.7)$ & $15(40.5)$ & $30(39.5)$ & $7(18.9)$ & 0.067 \\
\hline Male & $53(35.3)$ & $10(27.0)$ & $30(39.5)$ & $13(35.1)$ & 0.430 \\
\hline Smoking & $38(25.3)$ & $9(24.3)$ & $17(22.4)$ & $12(32.4)$ & 0.506 \\
\hline ECOG PS $\geq 2$ & $33(22.0)$ & $14(37.8)$ & $17(22.4)$ & $2(5.4)$ & 0.003 \\
\hline EGFR mutation & & & & & 0.049 \\
\hline Del 19 & $76(50.7)$ & $21(56.8)$ & $34(44.7)$ & $21(56.8)$ & \\
\hline L858R & $69(46.0)$ & $14(37.8)$ & $42(55.3)$ & $13(35.1)$ & \\
\hline Uncommon & $5(3.3)$ & $2(5.4)$ & $0(0)$ & $3(8.1)$ &
\end{tabular}

Burden of brain metastasis

\begin{tabular}{llllll}
\hline BM symptoms & $115(77.2)$ & $31(83.8)$ & $58(76.3)$ & $26(72.2)$ & 0.484 \\
\hline $\begin{array}{l}\text { BM maximal size, } \\
\text { Cm }\end{array}$ & $1.88(1.16)$ & $2.02(1.18)$ & $1.91(1.16)$ & $1.69(1.15)$ & 0.466 \\
\hline LM & $11(7.3)$ & $4(10.8)$ & $4(5.3)$ & $3(8.1)$ & 0.557 \\
\hline Lung-mol GPA $\geq 3$ & $72(48)$ & $16(43.2)$ & $36(47.4)$ & $20(54.1)$ & 0.640 \\
\hline Treatment & & & & & \\
\hline Anti-angiogenesis & $19(12.7)$ & $1(5.3)$ & $14(18.4)$ & $4(10.8)$ & 0.057 \\
\hline Local therapy modality & & & & \\
\hline \multicolumn{1}{|c|}{ WBRT } & $80(53.3)$ & $23(62.2)$ & $33(43.4)$ & $24(64.8)$ & 0.019 \\
\hline SRS & $37(24.6)$ & $7(18.9)$ & $23(30.2)$ & $7(18.9)$ & 0.273 \\
\hline Brain surgery & $46(30.7)$ & $10(27.0)$ & $22(28.9)$ & $14(37.8)$ & 0.643 \\
\hline PFS, months & $10.6(7.1-$ & $8.4(5.4-13.8)$ & $10.6(8.6-$ & $12.1(9.4-$ & 0.042 \\
\hline Osimertinib & $17.1)$ & & $18.9)$ & $18.4)$ & \\
\hline
\end{tabular}


BM: brain metastasis; ECOG PS: Eastern Cooperative Oncology Group performance status; EGFR: epidermal growth factor receptor; KPS: The Karnofsky Performance Scale. Lung-mol GPA: graded prognostic assessment for lung cancer with brain metastases using molecular markers; LM:

Leptomeningeal Metastases; PFS: progression-free survival; SRS: stereotactic radiosurgery; TKI: tyrosine kinase inhibitor; WBRT: whole-brain radiotherapy.

Continuous variables are presented as the mean (standard deviation) or median (interquartile range); categorical variables are presented as the number and percentage.

Table 2. Univariate and Multivariate Analysis of Clinical Factors Associated with Overall Survival

\begin{tabular}{lllllll} 
& \multicolumn{3}{c}{ Univariate } & & \multicolumn{1}{c}{$\begin{array}{c}\text { Multivariate } \\
\text { model }\end{array}$} \\
HR & $95 \% \mathrm{Cl}$ & $\begin{array}{l}p \\
\text { value }\end{array}$ & HR & $95 \% \mathrm{Cl}$ & $p$-value
\end{tabular}

$\begin{array}{lllllll}\text { Lung-mol GPA } \geq 3 & 0.561 & \begin{array}{lll}0.37- \\ 0.85\end{array} & 0.006 & 0.538 & \begin{array}{l}0.35- \\ 0.83\end{array} & 0.005\end{array}$

First-line EGFR-TKI

$\begin{array}{lll}\text { Erlotinib vs. Gefitinib } \begin{array}{lll}0.399 & 0.25- \\ 0.63\end{array} & <0.001\end{array}$

$\begin{array}{lll}\text { Afatinib vs. Gefitinib } \quad 0.387 & \begin{array}{l}0.22- \\ 0.66\end{array} & <0.001\end{array}$

$\begin{array}{llll}\text { Afatinib vs. Erlotinib } & 0.970 & 0.58- & 0.910 \\ 1.63\end{array}$

$\begin{array}{lllllll}\text { Non Gefitinib* vs. } & 0.395 & 0.26-0.60 & <0.001 & 0.521 & \begin{array}{l}0.33- \\ 0.82\end{array} & 0.004 \\ \text { Gefitinib } & & & & & 0.82\end{array}$

Local therapy

\begin{tabular}{|c|c|c|c|c|c|c|}
\hline SRS & 0.454 & $\begin{array}{l}0.28- \\
0.75\end{array}$ & 0.001 & 0.531 & $\begin{array}{l}0.32- \\
0.87\end{array}$ & 0.014 \\
\hline Brain surgery & 0.702 & $\begin{array}{l}0.45- \\
1.09\end{array}$ & 0.107 & - & - & - \\
\hline WBRT & 1.038 & $\begin{array}{l}0.69- \\
1.57\end{array}$ & 0.857 & - & - & - \\
\hline Anti-angiogenesis & 0.454 & $\begin{array}{l}0.21- \\
0.98\end{array}$ & 0.044 & 0.579 & $\begin{array}{l}0.27- \\
1.26\end{array}$ & 0.169 \\
\hline Osimertinib & 0.373 & $\begin{array}{l}0.22- \\
0.64\end{array}$ & $<0.001$ & 0.400 & $\begin{array}{l}0.23- \\
0.71\end{array}$ & 0.002 \\
\hline
\end{tabular}

Cl: confidence interval; EGFR: epidermal growth factor receptor; HR: hazard ratio; Lung-mol GPA: graded prognostic assessment for lung cancer with brain metastases using molecular markers; SRS: stereotactic 
radiosurgery; TKI: tyrosine kinase inhibitor; WBRT: whole-brain radiotherapy; Non Gefitinib*: Erlotinib or Afatinib

\section{Figures}

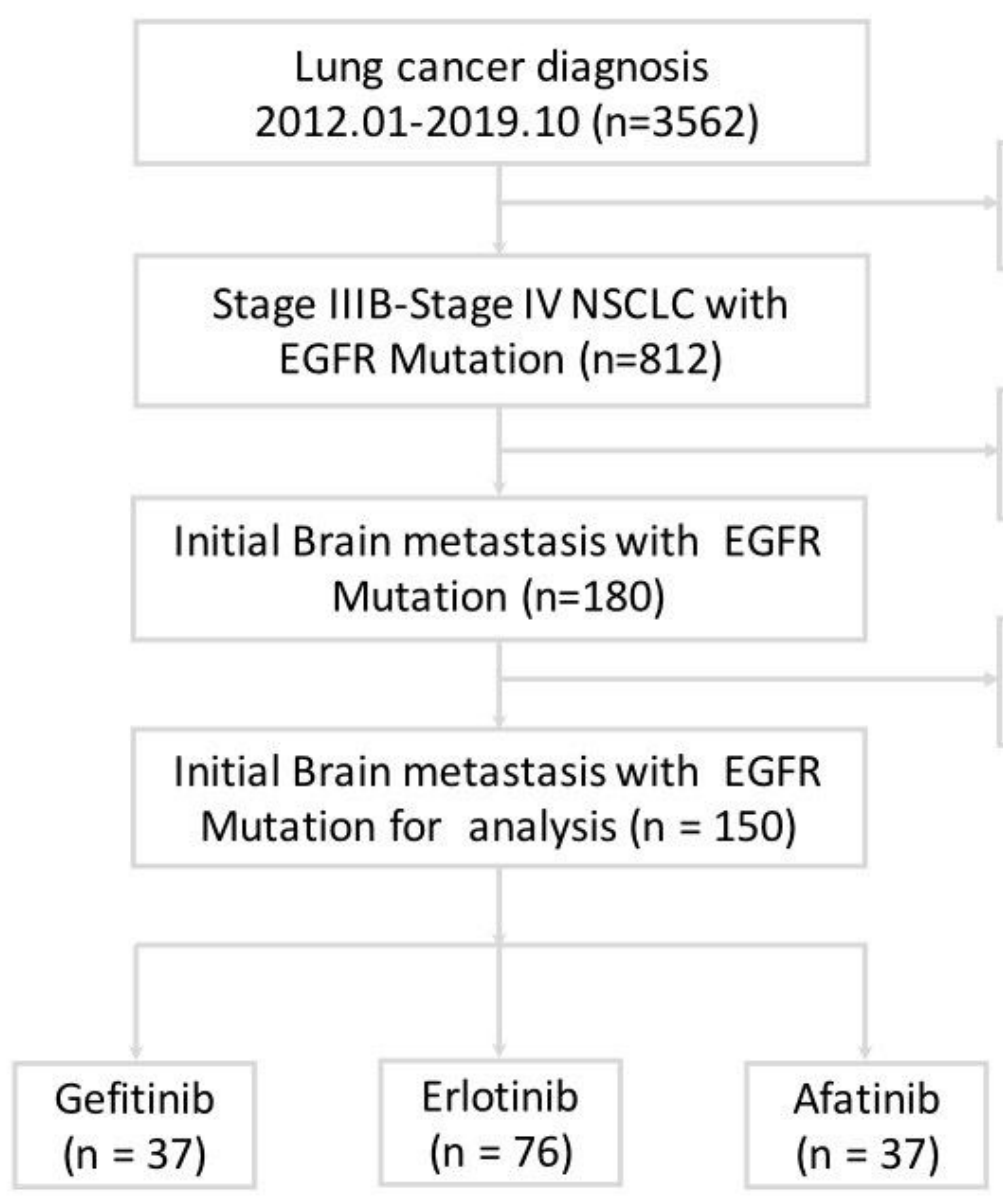

SCLC or NSCLC stage I-IIIA or EGFR wild type excluded $(n=2750)$

Initial no brain or no confirmation of brain metastasis excluded ( $n=632$ )

Insufficient data for analysis

excluded $(n=30)$

\section{Figure 1}

Flow diagram of patients meeting the eligibility criteria.

NSCLC: Non-small cell lung cancer; SCLC: Small cell lung cancer; EGFR: epidermal growth factor receptor 

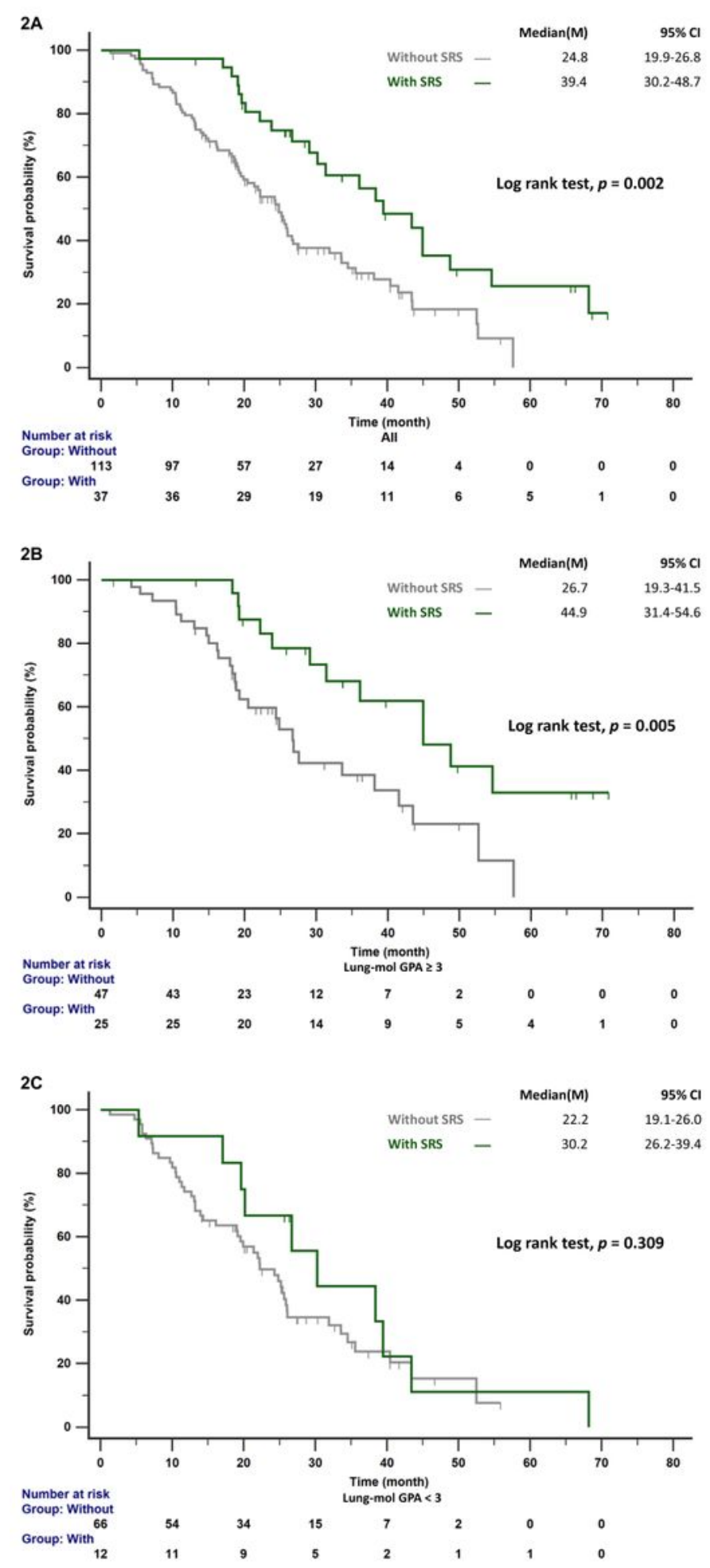

\section{Figure 2}

(A). Patients treated with SRS had increased median OS compared with those without SRS. (B). The median OS in patients with Lung-mol GPA $\geq 3$ who received EGFR-TKI plus SRS was longer than those who received EGFR-TKI without SRS. (C). No significant difference was observed in the median OS of patients with Lung-mol GPA $<3$ who received EGFR-TKI plus SRS and those who received EGFR-TKI without SRS. 
EGFR-TKI: epidermal growth factor receptor tyrosine kinase inhibitor; Lung-mol GPA: graded prognostic assessment for lung cancer using molecular markers; OS: overall survival; SRS: stereotactic radiosurgery
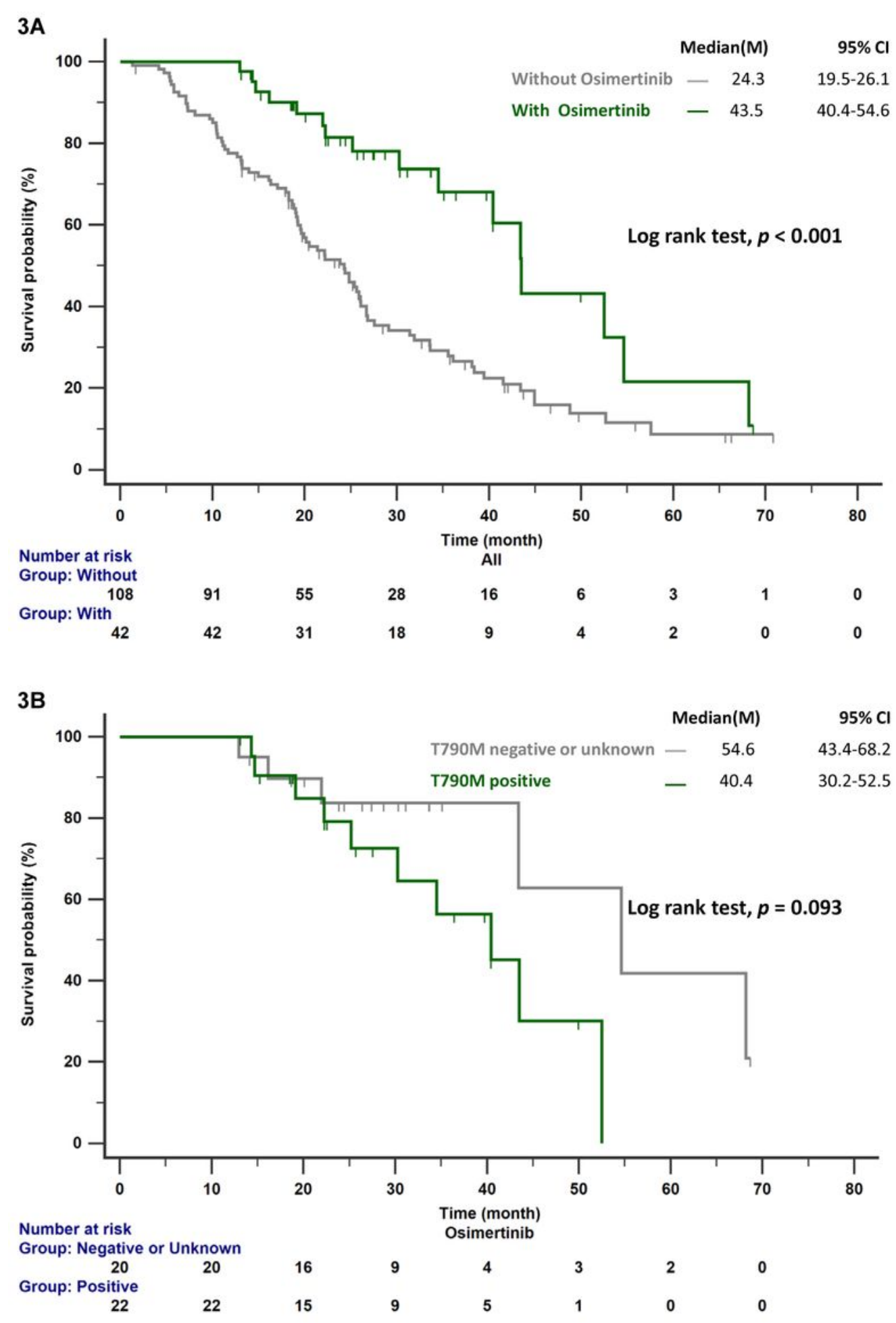

Figure 3

(A). The OS in patients who received sequential osimertinib was significantly longer than those without osimertinib treatment. (B). No significant difference in osimertinib treatment outcome was observed for 
patients with negative or unknown T790M status compared with patients with positive T790M.

OS: overall survival

\section{Figure 4}

Significantly longer survival was observed in patients with sequential osimertinib therapy, regardless of additional local brain therapy, compared with those without sequential osimertinib therapy.

Osi: osimertinib 